\title{
可见光促进的自由基型三重键插入反应在多取代芳(杂)环 合成中的应用
}

\author{
孙晓阳＼cjkstart俞寿云* \\ (南京大学化学化工学院 生命分析化学国家重点实验室 南京 210023)
}

\begin{abstract}
摘要 综述了本课题组近年来在利用可见光促进的自由基型三重键插入反应合成多取代芳香化合物方面的研究进展. 使用异腈插入反应构建了邻位取代吡啶、1-位取代异喹啉和 6-位取代的菲啶衍生物; 使用炔烃插入反应合成了多取代 的菜酚和呋喃及异喹啉酮衍生物; 还利用异腈/炔烃的多米诺插入反应构建了喹啉衍生物和异腈/氰基的多米诺插入反 应构建了喹喔啉衍生物. 这些反应都是在可见光促进的光氧化还原条件下进行, 反应条件温和, 产率较高.
\end{abstract}

关键词 光化学; 自由基; 炔烃; 异腈; 氰基; 芳香化合物

\section{Synthesis of Polysubstituted (Hetero)aromatic Compounds Using Visible-Light-Promoted Radical Triple Bond Insertions}

\author{
Sun, Xiaoyang Yu, Shouyun* \\ (State Key Laboratory of Analytical Chemistry for Life Science, School of Chemistry and Chemical Engineering, \\ Nanjing University, Nanjing 210023)
}

\begin{abstract}
Synthesis of polysubstituted (hetero)aromatic compounds enabled by visible-light-promoted radical triple bond insertions has been reviewed. A series of polysubstituted naphthols, furans and isoquinolones were prepared using radical alkyne insertions. 6-Substituted phenanthridine and 1-substituted isoquinoline and pyridine derivatives were synthesized assisted by radical isocyanide insertions. Furthermore, a wide scope of quinoline and quinoxaline derivatives were synthesized assisted by domino radical isocyanide/alkyne insertions and isocyanide/cyano insertions respectively. All reactions could be carried out under visible light photoredox catalysis with good to excellent chemical yields.
\end{abstract}

Keywords photochemistry; radical; alkyne; isocyanide; cyanide; aromatic compounds

芳香性化合物由于具有独特的生理活性和光电性 质 ${ }^{[1]}$, 因此被广泛地运用于药物分子和有机材料中, 所 以芳香性化合物的合成一直都是合成化学家重点关注 和研究的内容. 目前常见的多取代芳(杂)环的合成一般 采取芳香化合物直接取代的方式. 这种类型的反应一般 有以下几个缺点: 反应的区域选择性较差, 反应条件较 为苛刻, 合成步骤繁琐等. 利用官能团化的非环状前体 环化从头合成多取代芳(杂)环可以很好地克服以上缺 点. 该策略合成步骤较短, 往往是区域专一性的, 为合 成多取代的芳(杂)环提供了一种很好的可供选择的方 案.

近年来, 光敏催化作为一种简洁高效生成自由基的
方法，已被广泛应用于有机合成中 ${ }^{[2]}$. 我们课题组 ${ }^{[3 \sim 12]}$ 使用可见光促进的自由基型的三重键(包括炔烃，异腈 和腈)插入反应作为关键反应合成了一系列多取代的芳 (杂)环化合物, 包括菜酚、呋喃、吡啶、菲啶、异喹啉 酮、喹啉、异喹啉和喹喔啉等. 本文将对我们课题组最 近取得的进展进行总结, 并对相应的反应机理进行探 讨.

\section{1 自由基型异腈插入反应构建六元含氮芳杂环}

自从 Ugi 反应和其他相关的多组分反应被发现以 来, 异腈作为重要的合成模块被合成化学家广泛的应用 于有机合成中. 已有的研究主要集中在离子型异腈插入

\footnotetext{
*E-mail: yushouyun@nju.edu.cn

Received November 3, 2015; revised December 15, 2015; published online December 21, 2015.

Project supported by the National Natural Science Foundation of China (No. 21472084).

国家自然科学基金(No. 21472084)资助项目.
} 
(通常伴随着重排反应) ${ }^{[13]}$ 和过渡金属催化的异腈插入反 应 $^{[14]}$. 自由基型的异腈插入反应研究较少 ${ }^{[15]}$. 自由基型 的异腈插入反应由于具有反应温度一般是室温, 副产物 比较少, 反应速度较快的优点而被应用于含氮杂环的合 成. 我们课题组利用可见光促进的自由基型的异腈插入 反应构建了一系列的含氮六元芳杂环.

其反应原理如 Scheme 1 所示, 自由基前体 RX 被激 发态的光催化剂 $\mathrm{PC}^{*}$ 还原成自由基 $\mathrm{R}$, 然后加成到异腈 上, 生成亚胺(imidoyl)自由基中间体 I. 随后亚胺自由基 I 发生均裂芳香取代(homolytic aromatic substitution, HAS), 生成自由基中间体 II, 再经过氧化和去质子化, 得到一系列含氮杂环衍生物, 包括吡啶, 异喹啉和菲啶 等. $\mathrm{R}$ 取代基可以是烷基，芳基或者三氟甲基自由基 等 ${ }^{[15]}$.

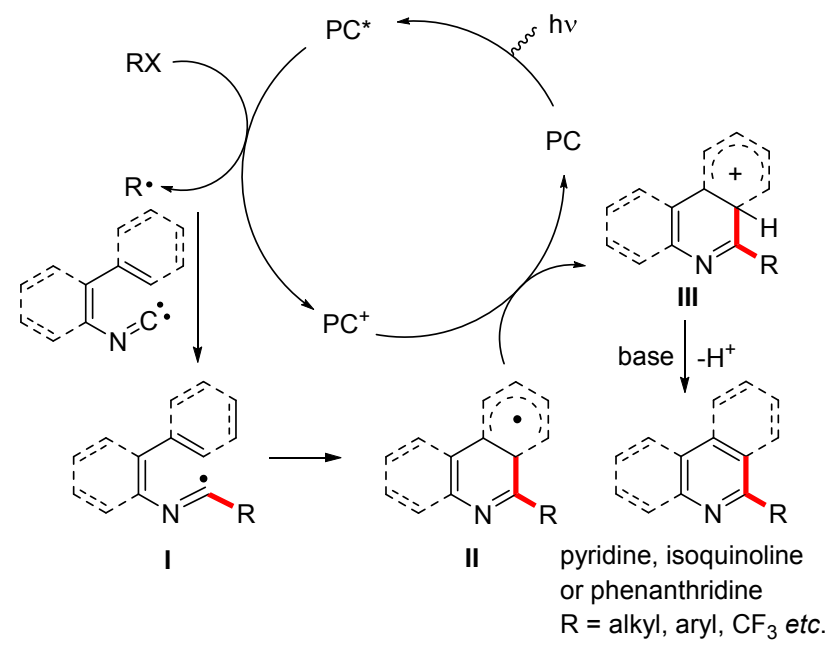

图式 1 自由基型异腈插入反应构建六元芳杂环推测机理

Scheme 1 Proposed mechanism for construction of six-numbered heteroaromatic rings by radical isocyanide insertions

\subsection{6 -取代菲啶衍生物的构建}

菲啶衍生物是一类重要的芳香化合物, 它们具有抗 癌、抗白血病、抗真菌、抗病毒的生理活性, 所以发展 高效菲啶衍生物的合成方法非常有意义. 我们基于可见 光促进的自由基异腈插入反应发展了一系列构建 6-取 代的菲啶的方法. 该方法无需当量的氧化剂或者毒性较 大的自由基前体, 一般只需要催化量的光催化剂, 在室 温条件下即可完成, 而且底物适用性较广, 产率较高.

\subsubsection{6-烷基菲啶环的构建}

2013 年, 我们课题组 ${ }^{[3]}$ 发展了第一例可见光诱导的 异腈的插入反应，并用此反应合成了一系列的 6-烷基化 的菲啶环衍生物. 我们选择联二芳基异腈 1 与溴化物 2 进行反应，在光催化剂的作用下很好的得到了产物 $\mathbf{3}$ (Eq. 1). 对于联二芳基异腈而言, 无论两个苯环是缺电 子还是富电子, 或者是杂环, 产率都很好 $(\mathbf{3 a} \sim 3 \mathbf{e})$. 对 于自由基前体溴化物而言, 适用性也很好, 产率也很高 $(\mathbf{3 f} \sim 3 \mathbf{3})$

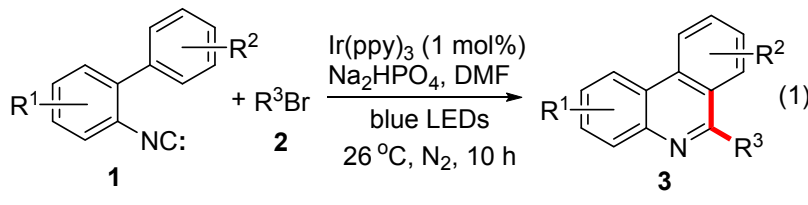

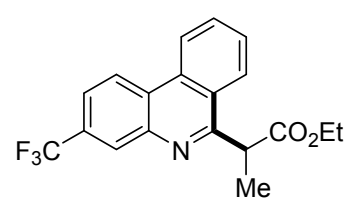

3a: $81 \%$

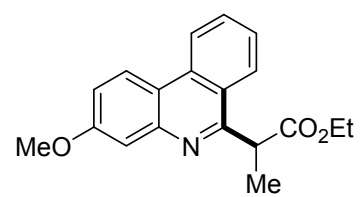

3b: $77 \%$<smiles>CCOC(=O)C(C)c1nc2ccccc2c2cc(OC(F)(F)F)ccc12</smiles>

3d: $75 \%$

3e: $63 \%$<smiles>CCCC(C(=O)OCC)c1nc2ccc(C)cc2c2ccccc12</smiles><smiles>Cc1ccc2nc(C3CCOC3=O)c3ccccc3c2c1</smiles>

3g: $81 \%$<smiles>CCOC(F)(F)c1nc2ccc(C)cc2c2ccccc12</smiles>

3h: $89 \%$<smiles>CCOC(=O)C(F)c1nc2ccc(C)cc2c2ccccc12</smiles>

3i: $68 \%$
6-烷基化菲定衍生物的成功构建给我们构建 6-一氟 或者二氟甲基化菲啶提供了很大的启发. 我们设想可以 将 $3 h$ 和 3i 的酯基水解然后脱羧得到 6-一氟或者二氟甲 基化的菲啶衍生物. 幸运的是，当我们将其水解后，将 反应液调至酸性，羧基即可直接脱除，以定量的收率得 到一氟或者二氟甲基化产物(Scheme 2) ${ }^{[4]}$.
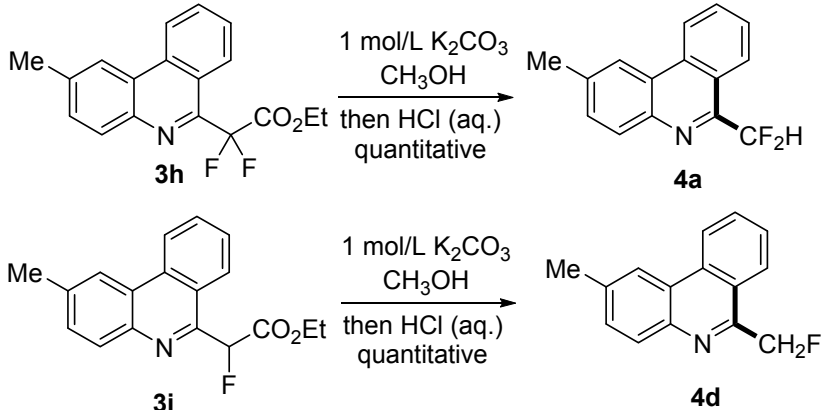

图式 2 室温条件下脱羰反应

Scheme 2 Room temperature decarboxylation

随后的底物的拓展表明该反应的产率和官能团容 忍度都很好. 为了进一步提高反应的效率, 我们 ${ }^{[4]}$ 将光 反应和水解脱羧在一锅中进行, 取得了与分步法相当的 产率(Eq. 2). 


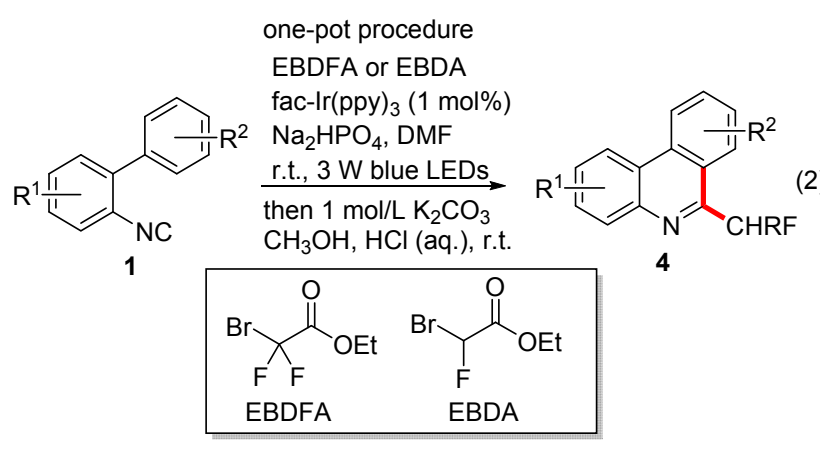<smiles>Cc1ccc2nc(C(F)F)c3ccccc3c2c1</smiles>

4a

One-pot: $64 \%$ Stepwise: $89 \%$<smiles>FC(F)c1nc2ccccc2c2ccc(-c3ccccc3)cc12</smiles>

$4 c$

One-pot: $66 \%$ Stepwise: $89 \%$<smiles>COc1ccc2c(c1)nc(CF)c1ccccc12</smiles>

$4 \mathrm{e}$

One-pot: $53 \%$ Stepwise: $64 \%$

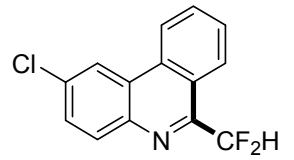

$4 \mathrm{~b}$

One-pot: $77 \%$

Stepwise: $73 \%$

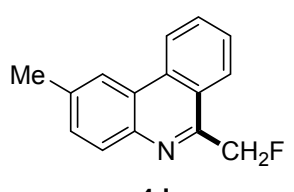

$4 d$

One-pot: $72 \%$

Stepwise: $67 \%$<smiles>COc1ccc2c(c1)nc(CF)c1cc(-c3ccccc3)ccc12</smiles>

One-pot: $54 \%$ Stepwise: $65 \%$

\subsubsection{6-三氟甲基菲啶环的构建}

三氟甲基的引入会显著地改变化合物的物理, 化学 和生物性质. 鉴于此, 我们课题组 ${ }^{[5,17]}$ 运用可见光诱导 的异腈插入反应构建了一系列 6-三氟甲基菲啶衍生物 (Eq. 3). 我们使用 Umemoto 试剂作为三氟甲基源, 钉的 配合物作为光催化剂, 非常成功地合成了结构多样的 6三氟甲基菲啶衍生物. 底物的适用性很好, 苯环的电性 对反应影响不大, 无论苯环上是给电子或者拉电子取代 基，产率都可以接受 $(\mathbf{5 a} \sim \mathbf{5 f})$.

有趣的是, 我们发现单纯依靠加热 $\left(60{ }^{\circ} \mathrm{C}\right)$ 也可以 从 Umemoto 试剂产生三氟甲基自由基，从而发生自由 基型的异腈插入反应，也可以以很好的收率合成 6-三氟 甲基化菲啶(Eq. 4, Scheme 3) ${ }^{[6]}$.

\subsubsection{6-芳基化菲啶环的构建}

在前面工作的基础上, 我们又发展了自由基型异腈 插入反应构建 6 -芳基化菲啶环的方法 ${ }^{[5,18]}$. 我们选取高 价芳基碘盐 6 作为芳基自由基前体, 在光催化剂的作用 下，非常高效地得到了一系列 6-芳基菲啶衍生物 7 (Eq.

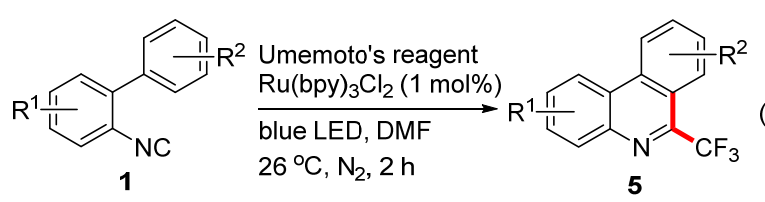<smiles>COc1ccc2c(c1)nc(C(F)(F)F)c1ccccc12</smiles>

5a: $77 \%$

5b: $75 \%$

5c: $85 \%$<smiles>COc1ccc2c(c1)c(C(F)(F)F)nc1ccccc12</smiles>

5d: $86 \%$<smiles>FC(F)(F)c1ccc2c(c1)nc(C(F)(F)F)c1ccccc12</smiles>

5e: $79 \%$<smiles>FC(F)(F)c1nc2ccccc2c2ccncc12</smiles>

5f. $55 \%$

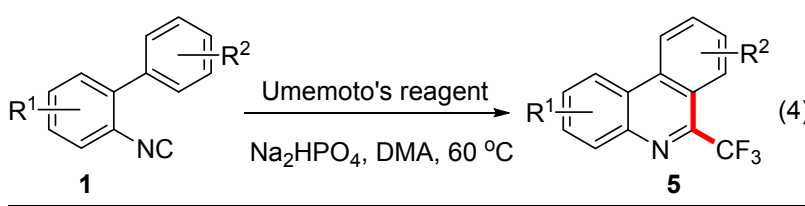<smiles>Cc1cc(C)c2nc(C(F)(F)F)c3ccccc3c2c1</smiles><smiles>Fc1ccc2nc(C(F)(F)F)c3ccccc3c2c1</smiles>
5g: $74 \%$

5h: $67 \%$<smiles>FC(F)(F)c1nc2ccccc2c2c3ccccc3c3ccccc3c12</smiles>

5j: $71 \%$

5k: $71 \%$

5I: $51 \%$

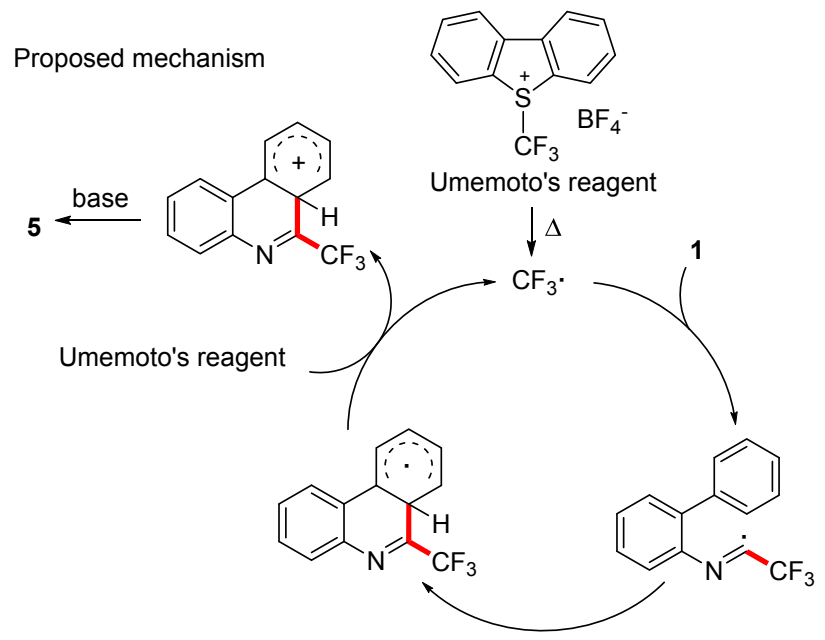

图式 3 自由基型异腈插入反应构建 6-三氟甲基取代菲啶环 推测机理

Scheme 3 Proposed mechanism for construction of 6-trifluoromethylated phenanthridines by radical isocyanide insertions 
5). 异腈的两个苯环以及高价碘化物的苯环上取代基的 变化对产率影响非常小 $(\mathbf{7 a} \sim 7 \mathbf{7 f})$.
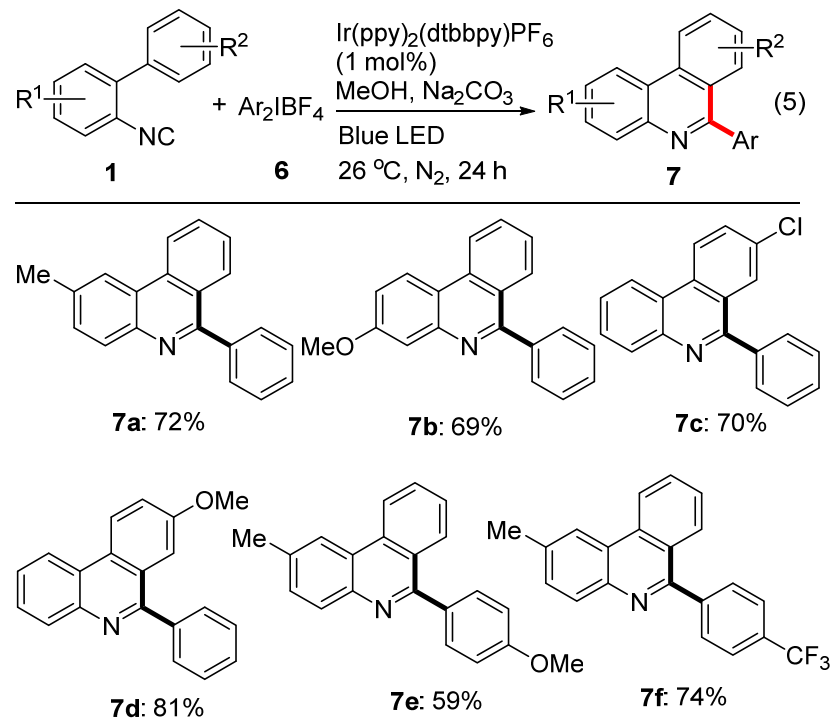

\subsection{1-取代异喹啉衍生物的构建}

异喹啉及其衍生物是天然产物, 生物活性分子和药 物分子的重要结构单元. 高效地合成这类化合物也是重 要的研究方向. 在成功构建 6-取代菲啶衍生物的基础 上, 我们课题组进一步将可见光促进的异腈的插入反应 运用到 1-取代异喹啉合成中. 目前异腈的化学主要使用 芳基或者烷基异腈作为主要的研究对象, 而烯基异腈的 研究非常少. 其原因可能是烯基异腈合成比较困难 ${ }^{[19]}$. 我们课题组改进了烯基异腈的合成方法，并报道了首例 可见光诱导的异腈插入反应构建 1-取代异喹啉衍生物.

\subsubsection{1-芳基化异喹啉环的构建}

烯基异腈 8 与芳基自由基前体高价芳基碘盐 $\mathbf{6}$ 在光 催化剂作用下反应，非常高效地合成了 1-芳基异喹啉衍 生物 9 (Eq. 6 $)^{[7,20]}$. 对于烯基异腈, 无论 $\mathrm{R}^{1}$ 是 $\mathrm{H}$ 、烷基或 者是芳基, 反应效果都非常好. 对于高价碘盐, 苯环上 无论是给电子或者拉电子取代基, 反应都能很顺利地进 行.

\subsubsection{1-三氟甲基化异喹啉环的构建}

在成功构建 1 -芳基化异喹啉环的基础上，我们 ${ }^{[8]}$ 又 成功地构建了 1-三氟甲基化的异喹啉(Eq. 7). Umemoto 试剂作为三氟甲基自由基前体, 无论对于三取代烯基异 腈还是四取代的烯基异腈, 反应效果都很好 (10a 10p). 对于四取代烯基异腈而言, $\mathrm{R}^{2}$ 既可以是芳基, 也可以是 烷基, 产率都比较高. 对于三取代烯基异腈, 产率也可 以令人接受.

\section{3 邻位取代吡啶衍生物的构建}

吡啶是最重要的芳香化合物之一. 合成取代的吡啶

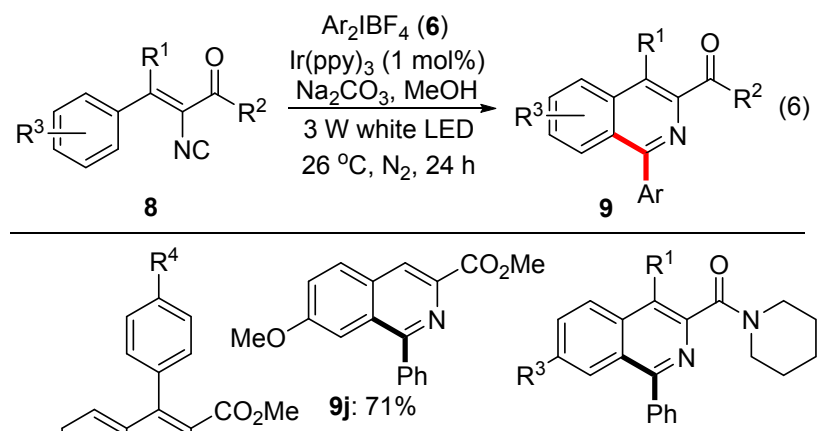

9k: $\mathrm{R}^{1}=\mathrm{Ph}, \mathrm{R}^{3}=\mathrm{H}, 83 \%$

9l: $\mathrm{R}^{1}=\mathrm{H}, \mathrm{R}^{3}=\mathrm{OMe}, 77 \%$

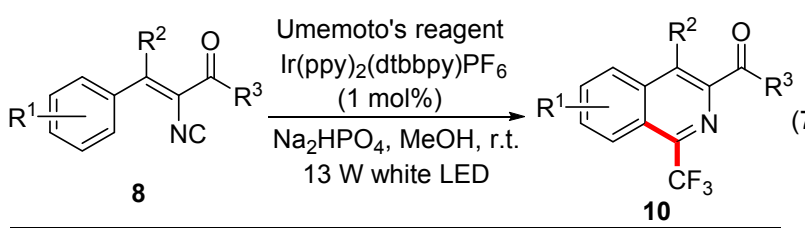

(c)

10a: $R^{1}=R^{4}=H, 94 \%$

10b: $R^{1}=R^{4}=\mathrm{Me}, 93 \%$

10c: $R^{1}=R^{4}=F, 91 \%$

10d: $R^{1}=R^{4}=$ OMe, $78 \%$

10e: $R^{1}=$ OMe, $R^{4}=H, 86 \%$

10f: $R^{1}=F, R^{4}=$ OMe, $80 \%$

10g: $R^{1}=$ OMe, $R^{4}=F, 87 \%$

10h: $\mathrm{R}^{1}=\mathrm{NO}_{2}, \mathrm{R}^{4}=\mathrm{H}, 52 \%$

10i: $R^{1}=H, R^{4}=F, 94 \%$

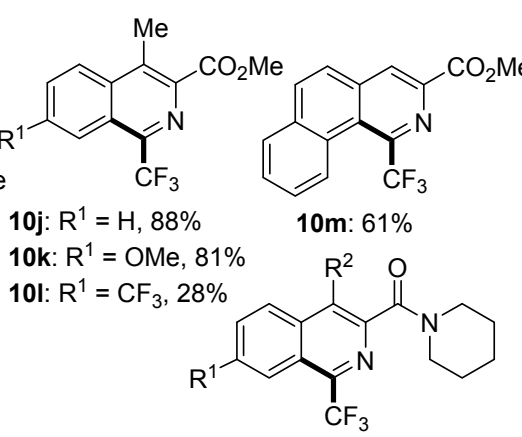

10n: $R^{1}=H, R^{2}=P h, 85 \%$

10o: $R^{1}=\mathrm{OMe}, \mathrm{R}^{2}=\mathrm{H}, 50 \%$<smiles>O=C(NCc1ccccc1)c1nc(C(F)(F)F)c2ccccc2c1-c1ccccc1</smiles>

也一直是合成化学家关注的重点. 区域专一性地合成取 代吡啶仍然是具有挑战性的课题. 最近我们课题组在成 功构建菲啶、异喹啉衍生物的基础上，发展了可见光促 进的区域专一性的邻位取代吡啶类衍生物的合成方 法 ${ }^{[9]}$.

我们以 Umemoto 试剂和烷基溴化物作为自由基的 前体，二烯基异腈作为自由基的受体，合成了一系列邻 位(氟)烷基化的吡啶衍生物(Eq. 8). 当以 Umemoto 试剂 
作为自由基前体, 反应的产率都很好 $(\mathbf{1 2 a} \sim 12 \mathrm{~h})$. 无论 $\mathrm{R}^{1}$ 是芳基、杂环、烷基或者是环状烷基，反应都可以很 顺利地进行. 当以烷基溴化物为自由基前体, 反应产率 也可以让人接受 $(12 \mathrm{i} \sim 12 \mathrm{p})$. 烷基溴化物可以是普通的 烷基溴化物或者含氟的烷基溴化物.<smiles>[R]C=C([R])C=C(C(C)=O)[N+](=O)[O-]</smiles>

11

12

Condition A: Umemoto reagent, fac-Ir(ppy) ${ }_{3}(1 \mathrm{~mol} \%), \mathrm{Na}_{2} \mathrm{HPO}_{4}$ $\mathrm{MeOH}$, r.t., 6 h, white LEDs<smiles>[R]C(=O)c1cc([N+](=O)[O-])c(-c2cc(C(=O)OC)nc(C(F)(F)F)c2-c2ccc(C(C)=O)nc2C(F)(F)F)c(C(C)=O)n1</smiles>

12a: $R^{3}=H, 77 \%$

12b: $R^{3}=\mathrm{OMe}, 66 \%$

12d: $48 \%$

12e: $48 \%$

12c: $R^{3}=\mathrm{CF}_{3}, 78 \%$<smiles>CC(=O)c1cc2c(c(C(F)(F)F)n1)CCCC2</smiles><smiles>CC(=O)c1cc2c3ccccc3n(C(=O)OC(C)(C)C)c2c(C(F)(F)F)n1</smiles>

12f: $51 \%$

12g: $56 \%$

12h: $80 \%$

Condition B: $\mathrm{RBr}$, fac-Ir(ppy $)_{3}(1 \mathrm{~mol} \%), \mathrm{Na}_{2} \mathrm{HPO}_{4}$, DMF, r.t., $12 \mathrm{~h}$, white LEDs<smiles>CCOC(C)c1nc(C(C)=O)cc(C)c1-c1ccccc1</smiles>

12i: $41 \%$<smiles>CCOC(OCC)c1nc(C(C)=O)cc(C)c1-c1ccccc1</smiles>

12k: $47 \%$<smiles>CCCC(C(=O)OCC)c1nc(C(C)=O)cc(C)c1-c1ccccc1</smiles>

12j: $57 \%$

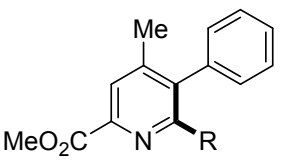

12I: $\mathrm{R}=\mathrm{CF}_{2} \mathrm{CO}_{2} \mathrm{Et}, 63 \%$

$12 \mathrm{~m}: \mathrm{R}=\mathrm{CFHCO}_{2} \mathrm{Et}, 53 \%$

12n: $\mathrm{R}=\mathrm{CF}_{2} \mathrm{SO}_{2} \mathrm{Ph}, 50 \%$

12o: $\mathrm{R}=\mathrm{C}_{6} \mathrm{~F}_{13}, 56 \%$

12p: $\mathrm{R}=\mathrm{CF}\left(\mathrm{CO}_{2} \mathrm{Et}\right)_{2}, 60 \%$

\section{2 自由基型炔烃插入反应构建多取代芳杂环化 合物}

荎酚和呋喃是天然产物和药物化学中重要的芳香 环体系. 鉴于其重要性, 我们发展了一种自由基型的炔 烃插入反应来构建多取代的荎酚和呋喃的方法 ${ }^{[10,21]}$. 其 反应原理如 Scheme 4 所示, 缺电子溴化物被激发态的 $\mathrm{Ir}^{\mathrm{II}}{ }^{*}$ 还原成烷基自由基 IV, 然后加成到炔烃上，生成烯 基自由基 $\mathrm{V}$ 或者 VI. 当 $\mathrm{R}^{1}$ 为芳基时, 烯基自由基 $\mathrm{V}$ 加 成到芳基上, 再经过氧化和去质子化, 得到多取代的䒺 酚. 当 $\mathrm{R}^{1}$ 为烷基时, 烯基自由基 $\mathrm{VI}$ 加成到羰基上, 再

经过氧化和去质子化，得到多取代的呋喃.

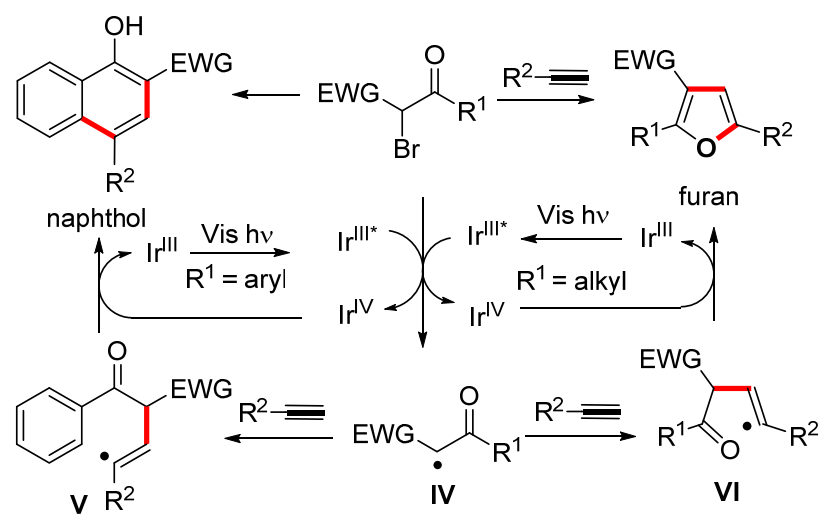

图式 4 自由基型炔烃插入反应构建多取代芳杂环推测机理 Scheme 4 Proposed mechanism for construction of polysubstitued heteroaromatic rings by radical alkyne insertions

\section{1 多取代萗酚环的构建}

我们选择苯酰乙酸酯的溴化物 13 和炔烃 14 作为起 始原料, 光催化剂为 $\operatorname{Ir}(\mathrm{ppy})_{3}$, 在白色 LED 灯带照射下 反应，生成一系列萗酚衍生物 ${ }^{[10]}$. 我们发现这个反应的 底物适用性非常广，产率也非常高(Eq. 9). 对于端炔而 言，不论是芳基端炔还是脂肪端炔，反应效果都很好 $(15 \mathrm{a} \sim 15 \mathrm{e})$. 对于溴化物而言，既可以是普通的芳基溴 化物也可以是杂环衍生的溴化物, 反应都可以很高效地 进行 $(\mathbf{1 5} \mathbf{f} \sim \mathbf{1 5} \mathbf{j})$. 非端炔的活性较低，需要将溴化物用 量增至 5 个当量, 才可以获得良好的产率 $(\mathbf{1 5 k})$.

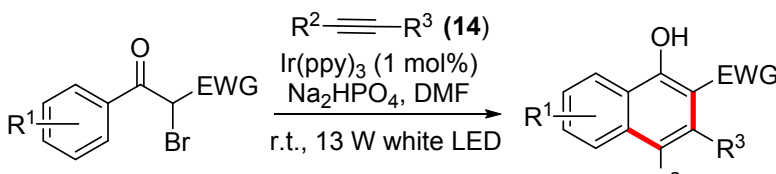

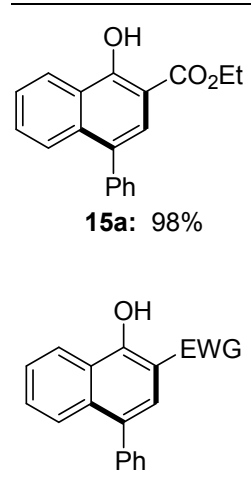

15g: $\mathrm{EWG}=\mathrm{CO}_{2} \mathrm{Bu}-t, 95 \%$ 15h: $\mathrm{EWG}=\mathrm{COPh}, 87 \%$ 15i: $\mathrm{EWG}=\mathrm{COMe}, 53 \%$

\section{2 多取代呋喃环的构建}

当选择 1,3-环己二酮衍生的溴化物 16 和炔烃作为 起始原料, 可以得到呋喃的衍生物 ${ }^{[10]}$. 在合成萘酚的反 应条件的基础上，通过摸索条件，我们只需把溶剂换成 
乙醇, 就可以得到较高产率的多取代呋喃衍生物. 随后 我们对底物进行了拓展, 反应可以很顺利的进行, 高效 的合成多取代的呋喃衍生物(Eq. 10). 对于芳基炔而言, 通过对芳环的电性进行调控, 我们发现芳环越富电产率 越高(17b), 相反芳环越缺电, 产率越低(17c). 相对复杂 的䒬炔也可以得到 $81 \%$ 产物(17d). 当在烷基溴化物上 面引入取代基 $\mathrm{R}^{1}$ 和 $\mathrm{R}^{2}$ 后, 反应仍然可以很顺利的进 行, 产率仍然非常高 $(\mathbf{1 7 e} \sim \mathbf{1 7 f})$.

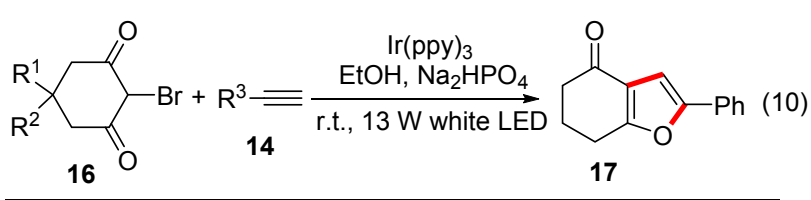

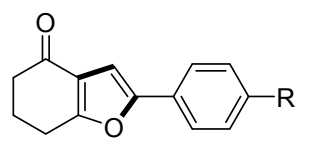

17a: $\mathrm{R}=\mathrm{H} \quad 60 \%$

17b: $\mathrm{R}=p$-OMe $\quad 96 \%$

17c: $\mathrm{R}=p-\mathrm{CF}_{3} \quad 33 \%$<smiles>[R]C1([R])CC(=O)c2cc(-c3ccc(OC)cc3)oc2C1</smiles>

$R^{2}$ 17e: $R^{1}=R^{2}=M e, 98 \%$

17f: $R^{1}=H, R^{2}=P h, 92 \%$
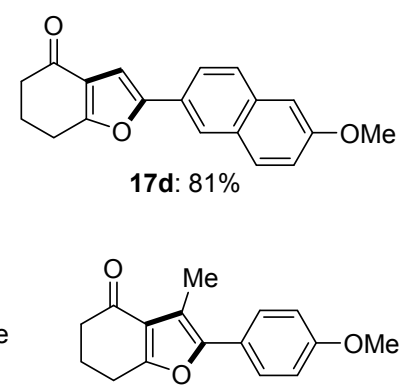

17g: $48 \%$

\section{3 多取代异喹啉酮环的构建}

异喹啉酮衍生物是许多天然产物和生物活性分子 的重要结构单元, 近年来如何高效合成异喹啉酮及其衍 生物吸引了许多有机合成化学家的注意. 最近我们课题 组 ${ }^{[11]}$ 发展了一种可见光促进的苯并三嗪酮脱氮气炔烃 插入方法高效构建异喹啉酮衍生物的方法, 具体反应机 制 Scheme 5 所示. 苯并三嗪酮被激发态 $\mathrm{Ir}^{\mathrm{III}}{ }^{*}$ 还原脱氮成 自由基氮负离子, 随后对炔烃加成生成中间体 VIII 或 者其共振式 IX. 自由基中间体 IX 对亚胺加成生成中间 体 $\mathbf{X}$ ，氧化后生成最终产物异喹啉酮.

随后进行的底物拓展表明苯并三嗪酮的两个芳香 环以及炔烃的芳香环的电性对反应的影响都很小 $(19 \mathrm{a} \sim 19 \mathrm{~s}, \mathrm{Eq} .11)$. 无论是给电子的芳环还是缺电子的 芳环，或者杂环炔烃，反应都可以很顺利地进行.

\section{3 自由基型异腈/炔烃多米诺插入反应构建喹 啉环}

喹啉骨架广泛存在于天然产物, 生物活性分子和药 物分子中, 对于有机化学家而言, 它们的合成是一项重 要的研究课题. 近年来, 我们课题组 ${ }^{[12,22]}$ 发展了一种可 见光诱导的异腈/炔烃多米诺插入反应构建喹啉的方法. 反应原理如 Scheme 6 所示, 缺电子炔烃溴化物被激发 态的 $\mathrm{Ir}^{\mathrm{II}}{ }^{*}$ 还原成烷基自由基, 然后加成到异腈上, 生成

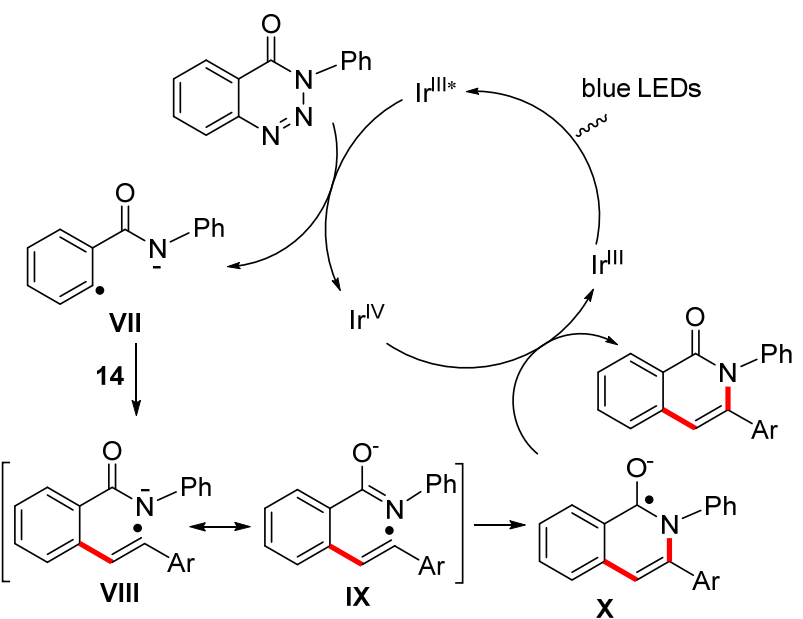

图式 5 自由基型炔烃插入反应构建异喹啉酮推测机理 Scheme 5 Proposed mechanism for construction of isoquinolones by radical alkyne insertions
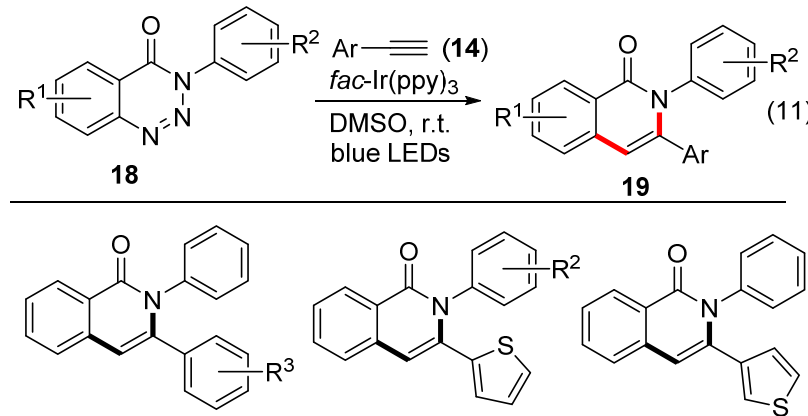

19a: $\mathrm{R}^{3}=p-\mathrm{MeO} \quad 83 \% \quad$ 19j: $\mathrm{R}^{2}=p$-OMe $\quad 64 \%$

19b: $\mathrm{R}^{3}=m-\mathrm{MeO} \quad 76 \%$

19c: $R^{3}=o-\mathrm{MeO} \quad 77 \%$

19d: $\mathrm{R}^{3}=p-\mathrm{F}$

19e: $\mathrm{R}^{3}=p-\mathrm{Cl}$

19f: $\mathrm{R}^{3}=p-\mathrm{Br}$

19g: $\mathrm{R}^{3}=p-\mathrm{Me} \quad 75 \%$

19h: $\mathrm{R}^{3}=p$-t-Bu $\quad 83 \%$

19i: $\mathrm{R}^{3}=p-\mathrm{Ph} \quad 54 \%$

19k: $\mathrm{R}^{2}=m$-OMe $\quad 68 \%$<smiles>O=c1c2ccccc2cc(-c2ccsc2)n1-c1ccccc1</smiles>

19I: $78 \%$<smiles>COc1ccccc1-n1c(-c2cccnc2)cc2ccccc2c1=O</smiles><smiles>[R][X]c1ccccc1-n1c(-c2ccccc2)cc2ccccc2c1=O</smiles>

19m: $50 \%$

9n: $\mathrm{R}^{2}=p-\mathrm{Me} \quad 75 \%$

19o: $\mathrm{R}^{2}=p-\mathrm{CF}_{3} \quad 72 \%$

19p: $\mathrm{R}^{2}=o-\mathrm{OMe} 72 \%$

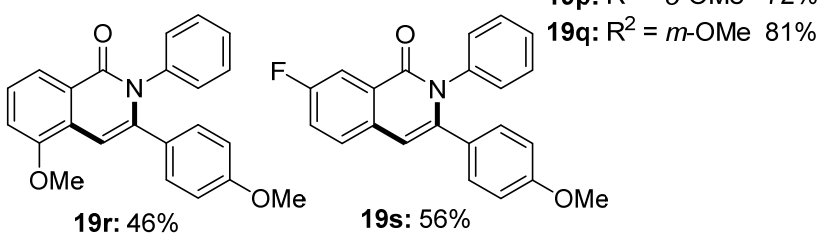

亚胺自由基 XI. 亚胺自由基 XI 对炔键加成得到烯基自 由基 XII，按路线 a 烯基自由基 XII 加成到芳基上，再经 过氧化和去质子化, 得到主产物的喹啉环衍生物; 按路 线 $\mathrm{b}$ 烯基自由基 XII 加成到芳基上生成螺环自由基中间 体 XV，然后开环生成氮自由基 XVI，氮自由基 XVI 对 苯环加成, 经过氧化和去质子, 得到异构体喹啉衍生物.

我们选芳基异腈 20 和炔烃溴化物 21 作为起始原料 在光敏催化剂催化下得到一系列喹啉衍生物 22 及其异 构体 22' (Eq. 12). 当 $\mathrm{R}^{1}$ 为 $\mathrm{Ph}$ 时, 能够分离得到少量的 区域异构体. 当 $\mathrm{R}^{1}$ 为其它基团时，未发现区域异构体的 


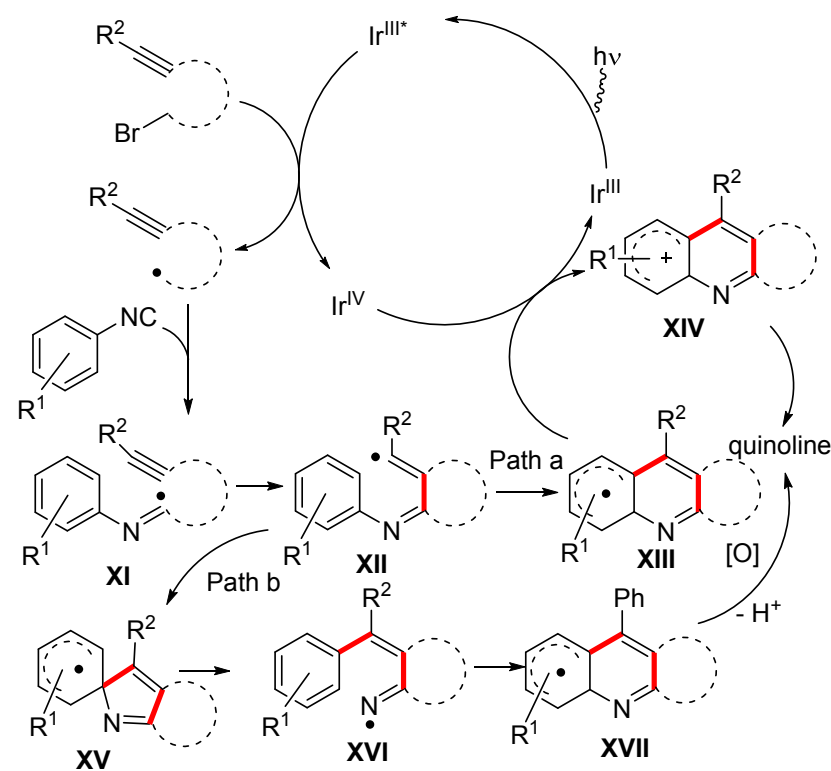

图式 6 自由基型异腈/炔烃多米诺插入反应构建喹啉环推测 机理

Scheme 6 Proposed mechanism for construction of quinolines by radical domino isocyanide/alkyne insertions

产生, 产率也非常高 $(\mathbf{2 2 b} \sim \mathbf{2 2 f})$. 此外 $\mathrm{R}^{2}$ 不仅可以是芳 基, 也可以是烷基，反应效果也非常好 $(\mathbf{2 2} \mathrm{g} \sim \mathbf{2 2 m})$.

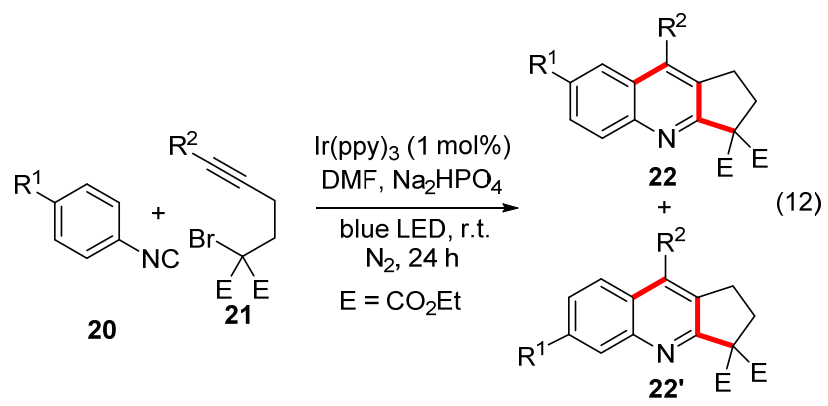<smiles>[R]c1ccc2nc3c(c(-c4ccccc4)c2c1)CCC3(F)F</smiles>

22a: $R^{1}=P h$, quant, ratio: $8.7: 1$

22b: $R^{1}=$ OMe, $96 \%$ 22c: $R^{1}=O P h, 91 \%$ 22d: $R^{1}=\mathrm{Cl}, 82 \%$ 22e: $\mathrm{R}^{1}=\mathrm{Br}, 85 \%$ 22f: $R^{1}=t-B u, 90 \%$<smiles>[R]c1c2c(nc3ccc(-c4ccccc4)cc13)C(F)(F)CC2</smiles>

22g: $\mathrm{R}^{2}=p-\mathrm{MeOC}_{6} \mathrm{H}_{4}, 95 \%$, ratio: $9.0: 1$ 22h: $\mathrm{R}^{2}=m-\mathrm{MeOC}_{6} \mathrm{H}_{4}$, quant, ratio: 10.8:1 22i: $\mathrm{R}^{2}=p-\mathrm{FC}_{6} \mathrm{H}_{4}$, quant, ratio: $6.3: 1$ 22j: $\mathrm{R}^{2}=p-\mathrm{ClC}_{6} \mathrm{H}_{4}$, quant, raito: $6.5: 1$ 22k: $R^{2}=p-t-B \mathrm{C}_{6} \mathrm{H}_{4}$, quant, ratio: 7.0:1 22I: $R^{2}=$ Me, $94 \%$, ratio: $4.6: 1$ 22m: $\mathrm{R}^{2}=n-\mathrm{C}_{5} \mathrm{H}_{11}, 92 \%$, ratio: $5.0: 1$

\section{4 自由基型异腈/氧基多米诺插入反应构建喹 喔啉环}

喹喔啉衍生物的合成一般是由邻二苯胺和二羰基 化合物, 环氧化物或者它们的等价物进行反应. 反应起 始原料的限制导致喹喔啉的合成仍然是一项非常具有
挑战性的课题. 我们在自由基型异腈/炔烃多米诺插入 反应构建喹啉环基础上发展了异腈/氰基的多米诺插入 反应，可以高效地构建喹喔啉衍生物 ${ }^{[12,23]}$. 其反应原理 如 Scheme 7 所示, 缺电子氰基溴化物被激发态的 $\mathrm{Ir}^{\mathrm{III}}$ 还原成烷基自由基，然后加成到异腈上，生成碳中心自 由基 XVIII. 自由基 XVIII 对氧基加成得到氮中心自由 基 XIX，氮中心自由基 XIX 加成到芳基上，再经过氧化 和去质子化, 得到最终的喹喔啉衍生物.

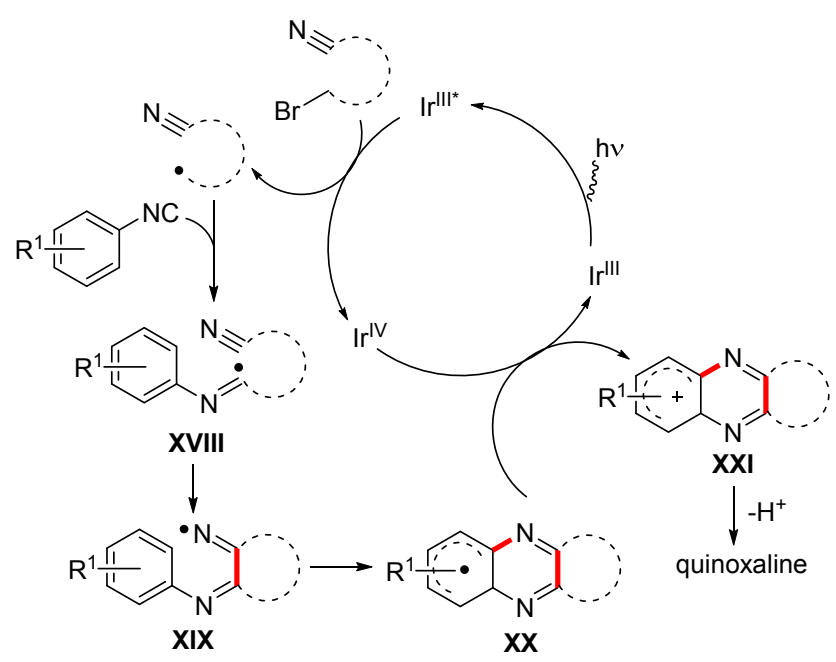

图式 7 自由基型异腈/氧基多米诺插入反应构建喹喔啉环推 测机理

Scheme 7 Proposed mechanism for construction of quinoxalines by radical domino isocyanide/cyanide insertions

我们选取芳基异腈 $\mathbf{2 0}$ 和氰基溴化物 $\mathbf{2 3}$ 作为起始原 料在光催化剂作用下反应, 很顺利的得到喹啉杂环 24 (Eq. 13). 底物的适用性较好, 官能团容忍性较高, 产率 中等以上 $(24 a \sim 241)$.

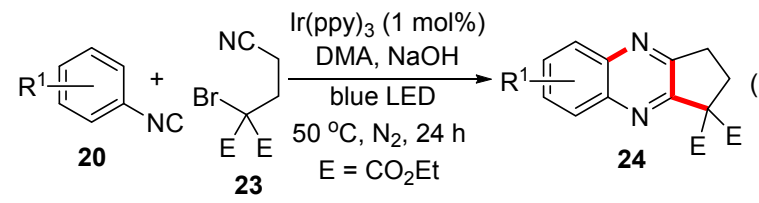<smiles>COc1cc2nc3c(nc2cc1OC)C(F)(F)CC3</smiles><smiles>FC1(F)CCc2nc3c[R11]ccc3nc21</smiles>

24b: $\mathrm{R}^{1}=p-\mathrm{Ph}, 76 \%$

24c: $\mathrm{R}^{1}=p-\mathrm{Cl}, 75 \%$

24d: $\mathbf{R}^{1}=p-\mathrm{Br}, 74 \%$

24e: $\mathrm{R}^{1}=p$-OMe, $71 \%$

24i: $74 \%$

24j: $65 \%$

24f: $\mathbf{R}^{1}=p$-OPh, $53 \%$

24g: $\mathrm{R}^{1}=p-t-\mathrm{Bu}, 73 \%$

24h: $\mathrm{R}^{1}=0-i-\mathrm{Pr}, 43 \%$<smiles>Cc1cc(C)c2nc3c(nc2n1)C(F)(F)CC3</smiles>

24k: $57 \%$

24I: $59 \%$ 


\section{5 结论与展望}

综上所述，我们课题组基于可见光促进的自由基型 三重键插入反应，发展了一系列构建多取代芳(杂)环的 合成新方法. 炔烃插入反应可以合成了多取代的萗酚, 呋喃和喹啉酮衍生物; 异腈插入反应可以构建了一系列 六元含氮芳杂环, 包括吡啶、异喹啉和菲啶衍生物; 异 腈/炔烃、异腈/氰基的多米诺插入反应分别构建了喹啉、 喹喔啉衍生物. 上述反应只需在光催化剂存在的情况 下, 通过可见光照射即可完成. 简单易操作, 反应温度 基本都是室温, 底物适用性非常广泛, 产率高, 对环境 友好. 这些成功的例子显示了可见光促进的三重键插入 反应合成芳(杂)环的强大威力. 可以预见, 该反应策略 可以用来构建其他的重要的(杂)芳香化合物, 比如苯、 吲哚、噁唑等.

\section{作者简介}

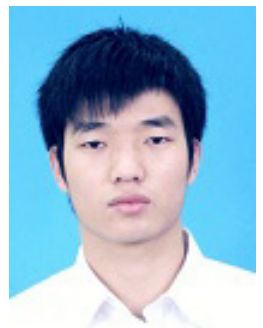

孙晓阳, 1989 年出生于中国河南. 2011 年于广西大 学获得理学学士学位. 同年进入南京大学化学化工学 院, 师从俞寿云副教授攻读博士学位. 主要研究方向是 可见光诱导的自由基化学.

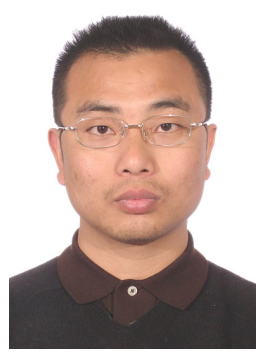

俞寿云博士, 南京大学化学化工学院副教授. 2001 年毕业于南京大学化学化工学院, 获得学士学位. 同年 进入中国科学院上海有机化学研究所攻读博士学位, 师 从马大为研究员, 2006 年 6 月获得博士学位后留所任助 理研究员. 2007 年 10 月至 2010 年 4 月, 在宾夕法尼亚 大学 Jeffrey Bode 教授小组进行博士后研究. 2010 年 9 月被聘为南京大学化学化工学院副教授, 课题组长. 主 要开展自由基化学和有机光化学以及具有生理活性天 然产物的全合成研究.

\section{References}

[1] (a) Evans, W. C. Nature 1977, 270, 17.

(b) Debnath, A. K.; Lopez de Compadre, R. L.; Debnath, G.; Shusterman, A. J.; Hansch, C. J. Med. Chem. 1991, 34, 786.

(c) Cornelisse, J.; Havinga, E. Chem. Rev. 1975, 75, 353.

(d) Claessens, C. G.; González-Rodríguez, D.; Torres, T. Chem. Rev. 2002, 102, 835 .

(e) Astruc, D. Tetrahedron 1983, 39, 4027.

[2] (a) Zeitler, K. Angew. Chem., Int. Ed. 2009, 48, 9785.

(b) Yoon, T. P.; Ischay, M. A.; Du, J. Nat. Chem. 2010, 2, 527.

(c) Narayanam, J. M. R.; Stephenson, C. R. J. Chem. Soc. Rev. 2011, 40, 102

(d) Shi, L.; Xia, W. Chem. Soc. Rev. 2012, 41, 7687.

(e) Xuan, J.; Xiao, W.-J. Angew. Chem., Int. Ed. 2012, 51, 6828.

(f) Tucker, J. W.; Stephenson, C. R. J. J. Org. Chem. 2012, 77, 1617.

(g) Prier, C. K.; Rankic, D. A.; MacMillan, D. W. C. Chem. Rev. 2013, 113, 5322.

(h) Hari, D. P.; König, B. Angew. Chem., Int. Ed. 2013, 52, 4734.

[3] Jiang, H.; Cheng, Y.; Wang, R.; Zheng, M.; Zhang, Y.; Yu, S. Angew. Chem., Int. Ed. 2013, 52, 13289.

[4] Sun, X.; Yu, S. Org. Lett. 2014, 16, 2938.

[5] Wang, R.; Jiang, H.; Cheng, Y.; Kadi, A. A.; Fun, H.-K.; Zhang, Y.; Yu, S. Synthesis 2014, 46, 2711

[6] Cheng, Y.; Jiang, H.; Zhang, Y.; Yu, S. Org. Lett. 2013, 15, 5520.

[7] Jiang, H.; Cheng, Y.; Wang, R.; Zhang, Y.; Yu, S. Chem. Commun. 2014, 50, 6164.

[8] Cheng, Y.; Yuan, X.; Jiang, H.; Wang, R.; Ma, J.; Zhang, Y.; Yu, S. Adv. Synth. Catal. 2014, 356, 2859.

[9] Tong, K.; Zheng, T.; Zhang, Y.; Yu, S. Adv. Synth. Catal. $\mathbf{2 0 1 5}, 357,3681$.

[10] Jiang, H.; Cheng, Y.; Zhang, Y.; Yu, S. Org. Lett. 2013, 15, 4884.

[11] Wang, H.; Yu, S. Org. Lett. 2015, 17, 4272.

[12] Sun, X.; Li, J.; Ni, Y.; Ren, D.; Hu, Z.; Yu, S. Asian J. Org. Chem. 2014, 3, 1317.

[13] (a) Zhu, J. Eur. J. Org. Chem. 2003, 1133.

(b) van Berkel, S. S.; Bögels, B. G. M.; Wijdeven, M. A.; Westermann, B.; Rutjes, F. P. J. T. Eur. J. Org. Chem. 2012, 3543.

(c) Dömling, A.; Ugi, I. Angew. Chem., Int. Ed. 2000, 39, 3168.

(d) Dömling, A. Chem. Rev. 2006, 106, 17.

[14] (a) Vlaar, T.; Ruijter, E.; Maes, B. U. W.; Orru, R. V. A. Angew. Chem., Int. Ed. 2013, 52, 7084.

(b) Qiu, G.; Ding, Q.; Wu, J. Chem. Soc. Rev. 2013, 42, 5257. (c) Lygin, A. V.; de Meijere, A. Angew. Chem., Int. Ed. 2010, 49, 9094.

(d) Lang, S. Chem. Soc. Rev. 2013, 42, 4867.

[15] (a) Zhang, B.; Studer, A. Chem. Soc. Rev. 2015, 44, 3505. (b) Wang, H.; Xu, B. Chin. J. Org. Chem. 2015, 35, 588.

[16] Other contributions on synthesis of aromatic rings using radical isocynide insertions:

(a) Xiao, T.; Li, L.; Lin, G.; Wang, Q.; Zhang, P.; Mao, Z.-W.; Zhou, L. Green Chem. 2014, 16, 2418.

(b) He, Z.; Bae, M.; Wu, J.; Jamison, T. F. Angew. Chem., Int. Ed. 2014, 53, 14451

(c) Gu, L.; Jin, C.; Liu, J.; Ding, H.; Fan, B. Chem. Commun. 
2014, 50, 4643 .

(d) Fu, W.; Zhu, M.; Xu, C.; Zou, G.; Wang, Z.; Ji, B. J. Fluorine Chem. 2014, 168, 50 .

[17] Other contributions on synthesis of 6-trifluoromethylated phenanthridines using radical isocynide insertions:

(a) Zhang, B.; Studer, A. Org. Lett. 2014, 16, 3990.

(b) Zhang, B.; Mück-Lichtenfeld, C.; Daniliuc, C. G.; Studer,

A. Angew. Chem., Int. Ed. 2013, 52, 10792.

(c) Wang, Q.; Dong, X.; Xiao, T.; Zhou, L. Org. Lett. 2013, 15,4846 .

[18] Other contributions to synthesis of 6-arylphenanthridines using radical isocynide insertions:

(a) Xia, Z.; Huang, J.; He, Y.; Zhao, J.; Lei, J.; Zhu, Q. Org. Lett. 2014, 16, 2546.

(b) Tobisu, M.; Koh, K.; Furukawa, T.; Chatani, N. Angew. Chem., Int. Ed. 2012, 51, 11363.

[19] Suzuki, M.; Nunami, K-I.; Matsumoto, K.; Yoneda, N.; Kasuga, O.; Yoshida, H.; Yamaguchi, T. Chem. Pharm. Bull. 1980, 28, 2374.

[20] Other contributions on synthesis of isoquinolines using vinyl isocyanides insertions:

(a) Zhang, B.; Studer, A. Org. Biomol. Chem. 2014, 12, 9895.

(b) Wang, H.; Yu, Y.; Hong, X.; Xu, B. Chem. Commun. 2014, 50, 13485.

(c) Qian, P.; Du, B.; Zhou, J.; Mei, H.; Han, J.; Pan, Y. RSC Adv. 2015, 5, 64961 .
[21] Other contributions to visible-light-promoted radical alkyne insertions:

(a) Yetiskin, O.; Dadashi-Silab, S.; Khan, S. B.; Asiri, A. M.; Yagci, Y. Asian J. Org. Chem. 2015, 4, 442.

(b) Tsuchii, K.; Doi, M.; Hirao, T.; Ogawa, A. Angew. Chem., Int. Ed. 2003, 42, 3490.

(c) Dong, X.; Xu, Y.; Liu, J. J.; Hu, Y.; Xiao, T.; Zhou, L. Chem.-Eur. J. 2013, 19, 16928.

[22] Other contributions on domino radical isocyanide/alkyne insertions:

(a) Josien, H.; Ko, S.-B.; Bom, D.; Curran, D. P. Chem.-Eur. J. 1998, 4, 67.

(b) Josien, H.; Curran, D. P. Tetrahedron 1997, 53, 8881.

(c) Curran, D. P.; Liu, H.; Josien, H.; Ko, S.-B. Tetrahedron 1996, 52, 11385.

(d) Curran, D. P.; Liu, H. J. Am. Chem. Soc. 1992, 114, 5863.

(e) Curran, D. P.; Du, W. Org. Lett. 2002, 4, 3215.

(f) Nanni, D.; Pareschi, P.; Tundo, A. Tetrahedron Lett. 1996, 37, 9337.

[23] Other contributions on domino radical isocyanide/cyanide insertions:

(a) Nanni, D.; Pareschi, P.; Rizzoli, C.; Sgarabotto, P.; Tundo, A. Tetrahedron 1995, 51, 9045 .

(b) Camaggi, C. M.; Leardini, R.; Nanni, D.; Zanardi, G. Tetrahedron 1998, 54, 5587. 